\title{
Chemoradiation with Weekly Paclitaxel and Carboplatin in Esophageal Squamous Cell Carcinoma: A Prospective Study
}

Deepa M. Joseph ${ }^{1}$ Monica Irukulla Malik² Jyothi Jonnadula² Fayaz Ahmed ${ }^{2}$ Deepthi Valiyaveettil ${ }^{2}$

${ }^{1}$ Department of Radiation Oncology, All India Institute of Medical Sciences, Rishikesh, Uttarakhand, India

${ }^{2}$ Department of Radiation Oncology, Nizam's Institute of Medical Sciences, Hyderabad, Telangana, India

South Asian J Cancer 2021;10:151-154.
Address for correspondence Monica Irukulla Malik, MD, Department of Radiation Oncology, Nizam's Institute of Medical Sciences, Hyderabad, Telangana 500082, India (e-mail: dr_monica11@yahoo.com).

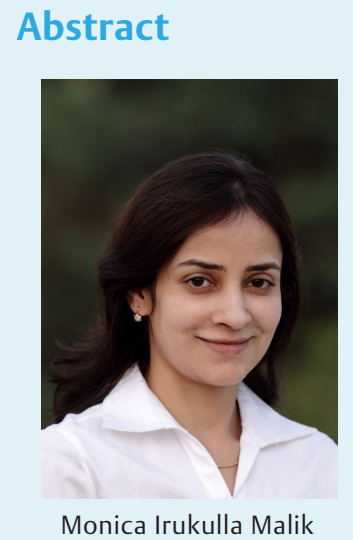

Keywords

- esophageal squamous cell carcinoma

- radical chemoradiation

- weekly paclitaxel and carboplatin
Objective Neoadjuvant chemoradiation (CRT) using paclitaxel and carboplatin has significantly improved the survival rates in carcinoma esophagus, especially in squamous cell carcinoma (SCC). This regimen has not been adequately explored prospectively as a definitive CRT strategy. Our aim was to evaluate the efficacy, toxicity, and compliance to this regimen in a prospective setting in locally advanced esophageal SCC. Materials and Methods Patients with locally advanced esophageal SCC were planned for definitive CRT by using weekly paclitaxel $50 \mathrm{mg} / \mathrm{m}^{2}$ and carboplatin area under curve 2 along with radical radiotherapy to a dose of 50.4 to 54 Gy. Treatment-related toxicity was assessed by using the common terminology criteria for Adverse Events Version 4.0, and the response was assessed by using endoscopy and computed tomography (CT) 4 to 6 weeks following CRT. The pathological response was documented for those who underwent surgery.

Results Fifteen patients were included in the study, and all patients completed the planned course of radiation. The median number of chemotherapy cycles received was four. In total, $66 \%$ of the patients had delay or interruptions in chemotherapy, mostly due to neutropenia, and $66 \%$ of the patients had a clinical complete response (CR). Four patients underwent definitive esophagectomy, and the histopathology revealed pathologic CR. Overall CR rate was $80 \%$. The median overall survival was 14 months, and 1 -year survival was $57 \%$.

Conclusion Definitive CRT in esophageal SCC using weekly paclitaxel and carboplatin was relatively well tolerated with manageable toxicities and good clinical response rates. It may potentially represent a new standard of care as definitive therapy in the management of these tumors.
DOI https://doi.org/10.1055/s-0041-1727067 ISSN 2278-330X

How to cite this article: Joseph D. M, Malik M. I, Jonnadula J, et al. Chemoradiation with weekly paclitaxel and carboplatin in esophageal squamous cell carcinoma: a prospective study. South Asian J Cancer 2021;10(3):151-154.
(C) 2021. MedIntel Services Pvt Ltd.

This is an open access article published by Thieme under the terms of the Creative Commons Attribution-NonDerivative-NonCommercial-License, permitting copying and reproduction so long as the original work is given appropriate credit. Contents may not be used for commercial purposes, or adapted, remixed, transformed or built upon. (https://creativecommons.org/licenses/by-nc-nd/4.0/).

Thieme Medical and Scientific Publishers Private Ltd A-12, Second Floor, Sector -2, NOIDA -201301, India 


\section{Introduction}

Esophageal cancer is the eighth most common cancer worldwide and sixth in mortality among all malignancies. ${ }^{1}$ Majority of the patients are diagnosed in advanced stages. Chemoradiation (CRT) plays an important role in the management of carcinoma esophagus: either as preoperative therapy or as definitive therapy in inoperable patients. Radiation dose of 41.4 to $54 \mathrm{~Gy}$ has been used in neoadjuvant or definitive settings. Historically, cisplatin and 5 fluorouracil (5 FU)-based chemotherapy has been used along with radiotherapy (RT) as the CRT regimen. Recent evidences suggest better survival, local control, and resection rates following paclitaxel- and carboplatin-based regimen. ${ }^{2}$

This study was done with an aim to assess the response to treatment and survival in patients treated with CRT by using weekly paclitaxel and carboplatin for esophageal carcinoma.

\section{Materials and Methods}

Patients aged between 18 and 75 years with histologically confirmed squamous cell carcinoma (SCC) of the esophagus or esophagogastric junction (Siewert I and II), TNM stage T1 to T4a, N0 to N2, M0, and a Karnofsky performance score of $\geq 70$ with adequate bone marrow reserve, renal and hepatic function, were included in the study. Patients with metachronous primary and those who received any previous treatment for any malignancy were excluded from the study. RT was delivered on linear accelerators by three-dimensional conformal RT technique. The target volume and critical organs were contoured on the plain CT simulation images fused with contrast-enhanced $\mathrm{CT}$ images and positron emission tomography images whenever available. The gross tumor volume (GTV) was contoured as the contrast-enhancing tumor along with endoscopy correlation. The clinical target volume (CTV) was contoured as GTV expanded by 3 to $4 \mathrm{~cm}$ superiorly and inferiorly along the length of the esophagus and cardia and $1-\mathrm{cm}$ radial expansion. The nodal CTV was contoured as 0.5 to $1.5 \mathrm{~cm}$ expansion from the nodal GTV. The celiac axis was covered for tumors of the distal esophagus or gastroesophageal junction. Supraclavicular nodes were included for the upper thoracic esophageal tumors. CTV was expanded by $0.5 \mathrm{~cm}$ to generate planning treatment volume (PTV). The boost PTV was created by 1 to $1.5 \mathrm{~cm}$ expansion from the initial GTV. Initial PTV was treated to a dose of 39.6 to $41.4 \mathrm{~Gy}$ at $1.8 \mathrm{~Gy}$ per fraction, followed by a cone-down boost to a total dose of 50.4 to $54 \mathrm{~Gy}$ in $1.8 \mathrm{~Gy}$ per fraction. Chemotherapy consisted of injection paclitaxel $50 \mathrm{mg} / \mathrm{m}^{2}$ and injection carboplatin area under the curve (AUC) 2 administered intravenously (IV) once weekly for five to six cycles along with RT.

All patients were monitored during the course of treatment with weekly blood counts and liver and kidney function tests. Other investigations were done as clinically indicated. Treatment-related toxicities were assessed by using the National Cancer Institute. Common terminology criteria for Adverse Events Version 4.0. Treatment response was assessed by using CT scan and endoscopy at 4 to 6 weeks of post-treatment.
The pathological response was documented for those who underwent surgery. Statistical analysis was done by using SPSS version 20 software (IBM SPSS, Chicago, IL). Survival analysis was done by using the Kaplan-Meier method.

\section{Results}

A total of 15 patients were included in the study. The median age of the study population was 55 years ranging from 35 to 70 years. The study included eight females (53\%) and seven males (47\%). Comorbidities included hypertension in two patients, coronary artery disease in two patients, and diabetes in one. One patient had hypothyroidism and two had albinism.

The median tumor length was $6.5 \mathrm{~cm}$ ranging from 3.4 to $10.8 \mathrm{~cm}$. Other patient and tumor characteristics are documented in -Table 1 . All patients underwent the planned course of RT without any significant interruptions. The median number of chemotherapy cycles received was four. Sixty-six percentage of the patients had delay or interruptions in chemotherapy, mostly due to neutropenia. Treatment-related toxicities are summarized in - Table 2 .

Two patients died within a month after CRT, one due to preexisting comorbidities, and the other patient developed metastatic disease. Sixty-six percentage of the patients had a clinical complete response (CR). Four patients underwent

Table 1 Patient characteristics

\begin{tabular}{|c|c|}
\hline Characteristic & Number (\%) \\
\hline Age (y), median (range) & $55(35-70)$ \\
\hline \multicolumn{2}{|l|}{ Sex, $n(\%)$} \\
\hline Males & $7(47)$ \\
\hline Females & $8(53)$ \\
\hline Tumor length (cm), median (range) & $6.5(3.4-10.8)$ \\
\hline \multicolumn{2}{|l|}{ Tumor location, $n(\%)$} \\
\hline Proximal third & $1(6)$ \\
\hline Middle third & $8(53)$ \\
\hline Distal third & $6(40)$ \\
\hline \multicolumn{2}{|l|}{ Clinical T-stage, $n(\%)$} \\
\hline cT1 & 0 \\
\hline cT2 & $1(6)$ \\
\hline cT3 & $9(60)$ \\
\hline cT4 & $5(33)$ \\
\hline \multicolumn{2}{|l|}{ Clinical N-stage, $n(\%)$} \\
\hline NO & $7(46)$ \\
\hline N1 & $4(26)$ \\
\hline N2 & $4(26)$ \\
\hline N3 & 0 \\
\hline \multicolumn{2}{|l|}{ KPS performance status score, $n$ (\%) } \\
\hline $80-90$ & $10(66)$ \\
\hline 70 & $5(33)$ \\
\hline
\end{tabular}

Abbreviation: KPS, Karnofsky Performance Score. 
Table 2 Incidence of grade 2 or more toxicity during chemoradiation

\begin{tabular}{|l|l|}
\hline Event & $\boldsymbol{n ( \% )}$ \\
\hline Anorexia & $2(13)$ \\
\hline Fatigue & $7(46)$ \\
\hline Vomiting & $7(46)$ \\
\hline Constipation & $3(20)$ \\
\hline Diarrhea & $3(20)$ \\
\hline Nausea & $3(20)$ \\
\hline Leukopenia & $11(73)$ \\
\hline
\end{tabular}

definitive esophagectomy, and the histopathology revealed a pathologic CR. Overall CR rate was $80 \%$. On follow-up, two patients developed progressive disease: one had liver metastasis and the other one supraclavicular metastasis. The median survival was 14 months, and overall survival (OS) at 1 year was $57 \%$.

\section{Discussion}

Historically, surgery was the mainstay of treatment for esophageal cancers. However, the outcomes were dismal with very poor survival rates. ${ }^{3}$ From 2001 to 2007, the overall 5-year survival rate for esophageal cancer was reported as $19 \%{ }^{4}$ RT alone also resulted in poor control rates with no long-term survival. ${ }^{5}$ CRT has been explored as a part of trimodality therapy with the aim of improving outcomes. One of the most important initial experiences with CRT for esophageal cancer was described by Herskovic et al. ${ }^{6}$ Their protocol delivered radiation to a large field in $2 \mathrm{~Gy}$ fractions to a total dose of $30 \mathrm{~Gy}$ along with concomitant 5-FU/cisplatin chemotherapy, followed by a $20 \mathrm{~Gy}$ boost to a smaller field along with mitomycin $\mathrm{C}$ and bleomycin, which was later replaced with 5 FU and cisplatin. This small study of 22 patients showed a median survival of 22 months. This became the basis for the phase III RTOG 8501 trial comparing RT alone versus CRT, which showed a survival advantage with combined modality arm. ${ }^{5}$ The RT fields in the CRT arm included the entire esophagus in the initial $30 \mathrm{~Gy}$ and a boost of $20 \mathrm{~Gy}$ to $5 \mathrm{~cm}$ above and below the demonstrable tumor. Subsequently, the intergroup 0123 trial used a smaller RT field of $5 \mathrm{~cm}$ above and below the tumor and $2 \mathrm{~cm}$ radial margin. This trial established the radiation dose of $50.4 \mathrm{~Gy}$ in $1.8 \mathrm{~Gy}$ per fraction as the standard dose in CRT of carcinoma esophagus. ${ }^{7}$

Two phase III randomized trials have examined definitive CRT against CRT followed by surgery. ${ }^{8,9}$ Both these trails did not report any significant difference in OS. The French FFCD 9102 trial using 5 FU and cisplatin reported higher local control (65 vs. $57 \%, p<0.05$ ), and fewer stents required (5 vs. $32 \%, p<0.001)$ trimodality arm compared with CRT. In the German trial, there was a higher rate of 2-year freedom from local progression (64 vs. $41 \%, p=0.003$ ) when the surgery was added. This trial used cisplatin and etoposide as concurrent chemotherapy.
Multiple phase II studies have evaluated paclitaxel-based chemoradiotherapy regimens in esophageal cancerwith results comparable to 5 FU-based regimen with lower toxicity. ${ }^{10,11}$ The standard treatment of operable esophageal cancer now is trimodality treatment using neoadjuvant CRT followed by surgery. CALGB 9781 randomly assigned 56 patients to either esophagectomy with node dissection alone or CRT followed by esophagectomy with node dissection. CRT consisted of cisplatin $100 \mathrm{mg} / \mathrm{m}^{2}$ and FU $1000 \mathrm{mg} / \mathrm{m}^{2} / \mathrm{d}$ for 4 days on weeks 1 and 5 concurrent with radiation therapy (50.4 Gy total: $1.8 \mathrm{~Gy} /$ fraction over 5.6 weeks). This trial was closed due to poor accrual. Five-year survival was $39 \%$ versus $16 \%$ in favor of trimodality therapy..$^{12}$ A meta-analysis by Gebski et al also showed a survival advantage of neoadjuvant CRT. ${ }^{13}$

Recently, results of two large phase III randomized trials have been published regarding the use of neoadjuvant CRT with conflicting outcomes. ${ }^{2,14}$ The French trial included 195 patients from 30 centers randomly assigned to surgery versus CRT followed by surgery. RT dose was $45 \mathrm{~Gy}$ in 25 fractions over 5 weeks with two courses of concomitant chemotherapy composed of FU $800 \mathrm{mg} / \mathrm{m}^{2}$ and cisplatin $75 \mathrm{mg} / \mathrm{m}^{2}$. Compared with surgery alone, neoadjuvant CRT with cisplatin plus FU did not improve R0 resection rate or survival but enhanced postoperative mortality in patients with stage I or II esophageal cancers. The ChemoRadiotherapy for Oesophageal cancer followed by Surgery Study (CROSS) trial included 368 patients randomized between neoadjuvant chemoradiotherapy plus surgery versus surgery alone. CRT consisted of $41.4 \mathrm{~Gy}$ in $1.8 \mathrm{~Gy}$ per fraction along with paclitaxel $50 \mathrm{mg} / \mathrm{m}^{2}$ and carboplatin AUC 2 administered IV weekly. After a median follow-up of 84.1 months, there was a significant improvement in median OS (48.6 vs. 24 months) and $\mathrm{R} 0$ resection rates in those receiving neoadjuvant CRT. It was noteworthy that a greater benefit was observed in patients with SCC versus adenocarcinoma (median OS 81.6 vs. 43.2 months).

There are no large prospective series using paclitaxel and carboplatin in definitive CRT in carcinoma esophagus. There are few reports of small series using this regimen in definitive CRT. ${ }^{15-17}$ In a retrospective analysis by Noronha et al, the median progression free survival was 11 months and median OS was 19 months with manageable toxicity..$^{18}$ All patients in our study were able to receive the planned dose of radiation of 50.4 to $54 \mathrm{~Gy}$. Majority of our patients achieved complete remission with CRT using weekly paclitaxel and carboplatin. All the patients who underwent surgery had a complete pathological response. This demonstrates that this regimen is highly effective with a good clinical and pathological response and may obviate the need for subsequent surgery, especially in SCC.

\section{Conclusion}

Emerging evidence suggests that CRT may be the most effective treatment strategy for locally advanced SCC of the esophagus. CRT using weekly paclitaxel and carboplatin 
was relatively well tolerated and resulted in a high $C R$ rate in locally advanced SCC of the esophagus. It may potentially represent a new standard of care as definitive therapy in the management of these tumors.

\section{Funding \\ None.}

\section{Conflict of Interest}

None declared.

\section{References}

1 Ferlay J, Soerjomataram I, Dikshit R, et al. Cancer incidence and mortality worldwide: sources, methods and major patterns in GLOBOCAN 2012. Int J Cancer 2015;136(5):E359-E386

2 Shapiro J, van Lanschot JJ, Hulshof MCC, et al; CROSS study group. Neoadjuvant chemoradiotherapy plus surgery versus surgery alone for oesophageal or junctional cancer (CROSS): long-term results of a randomised controlled trial. Lancet Oncol 2015;16(9):1090-1098

3 D'Amico TA. Outcomes after surgery for esophageal cancer. Gastrointest Cancer Res 2007;1(5):188-196

4 Siegel RL, Miller KD, Jemal A. Cancer statistics, 2016. CA Cancer J Clin 2016;66(1):7-30

5 Herskovic A, Martz K, al-Sarraf M, et al. Combined chemotherapy and radiotherapy compared with radiotherapy alone in patients with cancer of the esophagus. N Engl J Med 1992; 326(24):1593-1598

6 Herskovic A, Leichman L, Lattin P, et al. Chemo/radiation with and without surgery in the thoracic esophagus: the Wayne State experience. Int J Radiat Oncol Biol Phys 1988;15(3):655-662

7 Minsky BD, Pajak TF, Ginsberg RJ, et al. INT 0123 (Radiation Therapy Oncology Group 94-05) phase III trial of combined-modality therapy for esophageal cancer: highdose versus standard-dose radiation therapy. J Clin Oncol 2002;20(5):1167-1174

8 Bedenne L, Michel P, Bouché $\mathrm{O}$, et al. Chemoradiation followed by surgery compared with chemoradiation alone in squamous cancer of the esophagus: FFCD 9102. J Clin Oncol 2007;25(10):1160-1168

9 Stahl M, Stuschke M, Lehmann N, et al. Chemoradiation with and without surgery in patients with locally advanced squamous cell carcinoma of the esophagus.JClin Oncol 2005;23(10):2310-2317

10 Kim DW, Blanke CD, Wu H, et al. Phase II study of preoperative paclitaxel/cisplatin with radiotherapy in locally advanced esophageal cancer. Int J Radiat Oncol Biol Phys 2007;67(2):397-404

11 Kelsey CR, Chino JP, Willett CG, et al. Paclitaxel-based chemoradiotherapy in the treatment of patients with operable esophageal cancer. Int J Radiat Oncol Biol Phys 2007;69(3):770-776

12 Tepper J, Krasna MJ, Niedzwiecki D, et al. Phase III trial of trimodality therapy with cisplatin, fluorouracil, radiotherapy, and surgery compared with surgery alone for esophageal cancer: CALGB 9781. J Clin Oncol 2008;26(7):1086-1092

13 Gebski V, Burmeister B, Smithers BM, Foo K, Zalcberg J, Simes J; Australasian Gastro-Intestinal Trials Group. Survival benefits from neoadjuvant chemoradiotherapy or chemotherapy in oesophageal carcinoma: a meta-analysis. Lancet Oncol 2007;8(3):226-234

14 Mariette C, Dahan L, Mornex F, et al. Surgery alone versus chemoradiotherapy followed by surgery for stage I and II esophageal cancer: final analysis of randomized controlled phase III trial FFCD 9901. J Clin Oncol 2014;32(23):2416-2422

15 Honing J, Smit JK, Muijs CT, et al. A comparison of carboplatin and paclitaxel with cisplatinum and 5-fluorouracil in definitive chemoradiation in esophageal cancer patients. Ann Oncol 2014;25(3):638-643

16 Haj Mohammad N, Hulshof MC, Bergman JJ, et al. Acute toxicity of definitive chemoradiation in patients with inoperable or irresectable esophageal carcinoma. BMC Cancer 2014;14:56

17 Ruppert BN, Watkins JM, Shirai K, et al. Cisplatin/Irinotecan versus carboplatin/paclitaxel as definitive chemoradiotherapy for locoregionally advanced esophageal cancer. Am J Clin Oncol 2010;33(4):346-352

18 Noronha V, Prabhash $\mathrm{K}$, Joshi $\mathrm{A}$, et al. Clinical outcome in definitive concurrent chemoradiation with weekly paclitaxel and carboplatin for locally advanced esophageal and junctional cancer. Oncol Res 2016;23(4):183-195 